\title{
Anatomic and functional imaging in the diagnosis of spine metastases and response assessment after spine radiosurgery
}

\author{
Moaaz Soliman, BS, ${ }^{1}$ Neil K. Taunk, MD, MS, ${ }^{2}$ Robert E. Simons, BS, ${ }^{1}$ Joseph R. Osborne, MD, PhD, ${ }^{3}$ \\ Michelle M. Kim, MD, ${ }^{1}$ Nicholas J. Szerlip, MD, ${ }^{4}$ and Daniel E. Spratt, MD ${ }^{1}$ \\ Departments of ${ }^{1}$ Radiation Oncology and ${ }^{4}$ Neurosurgery, University of Michigan Cancer Center, Ann Arbor, Michigan; and \\ Departments of ${ }^{2}$ Radiation Oncology and ${ }^{3}$ Radiology, Memorial Sloan Kettering Cancer Center, New York, New York \\ Spine stereotactic radiosurgery (SSRS) has recently emerged as an increasingly effective treatment for spinal metas- \\ tases. Studies performed over the past decade have examined the role of imaging in the diagnosis of metastases, as \\ well as treatment response following SSRS. In this paper, the authors describe and review the utility of several imaging \\ modalities in the diagnosis of spinal metastases and monitoring of their response to SSRS. Specifically, we review the \\ role of CT, MRI, and positron emission tomography (PET) in their ability to differentiate between osteoblastic and osteo- \\ lytic lesions, delineation of initial bony pathology, detection of treatment-related changes in bone density and vertebral \\ compression fracture after SSRS, and tumor response to therapy. Validated consensus guidelines defining the imaging \\ approach to SSRS are needed to standardize the diagnosis and treatment response assessment after SSRS. Future \\ directions of spinal imaging, including advances in targeted tumor-specific molecular imaging markers demonstrate early \\ promise for advancing the role of imaging in SSRS.
}

https://thejns.org/doi/abs/10.3171/2016.9.FOCUS16350

KEY WORDS spine metastases; treatment response; imaging; stereotactic body radiotherapy

$\mathrm{R}$ ECENT estimates show that approximately 280,000 adults in the United States are living with a diagnosis of metastatic bone disease, ${ }^{26}$ and an estimated $10 \%$ of cancer patients develop symptomatic spinal metastases. ${ }^{36}$ Nearly $40 \%$ of patients with metastatic cancer will have disease in the spine at the time of death, making the spine the most common site of osseous metastatic disease. ${ }^{59}$ If left undetected and untreated, spine disease can lead to severe pain secondary to pathologic fracture or catastrophic neurological compromise where there is epidural spinal cord compression.

Spinal metastases are typically diagnosed using CT, MRI, and/or positron emission tomography (PET) in conjunction with the presence of concerning signs and symptoms. The accuracy of each of these modalities in diagnosing skeletal metastasis has been examined in several meta-analyses using a combination of histopathological analysis, close clinical imaging and follow-up, and/or radiographic confirmation with multiple imaging techniques as the reference standard. ${ }^{18}$ The goal of diagnostic imaging in suspected spinal metastasis is to determine both the presence of malignant bone disease and the extent of malignant disease. Choice of diagnostic imaging modality is guided by the need for early diagnosis and gathering clinical information for comparison with post-treatment imaging. Information such as the presence of spinal cord compression, pathologic fractures, bone density, and skeletal disease burden plays an important role in the assessment of initial disease. Barriers to early diagnosis must be considered, however, given the clinical context. These challenges vary by modality and include cost, availability, soft tissue resolution, and whole skeletal assessment. Nevertheless, it is important to understand recent advances in diagnostic imaging tools, as they have improved the ability to diagnose spinal metastases early and to collect the necessary clinical information to guide treatment decisions.

Surgery and radiation therapy (RT) are currently the primary local treatments for spine disease, and when com-

ABBREVIATIONS ADC = apparent diffusion coefficient; DCE-MRI = dynamic contrast-enhanced MRI; DWI = diffusion-weighted imaging; MDA = MD Anderson; PET = positron emission tomography; RECIST = Response Evaluation Criteria in Solid Tumors; RT = radiation therapy; SPINO = Spine Response Assessment in Neuro-Oncology; SSRS = spine stereotactic radiosurgery; STIR = short tau inversion recovery; VCF = vertebral compression fracture.

SUBMITTED August 22, 2016. ACCEPTED September 14, 2016.

INCLUDE WHEN CITING DOI: 10.3171/2016.9.FOCUS16350.

* Mr. Soliman and Dr. Taunk contributed equally to this work. 
bined, they may be more effective than either modality alone. ${ }^{30,35}$ Conventional RT, consisting of daily low-dose radiation fractions, has been the mainstay in the treatment of spinal metastasis. ${ }^{27}$ Spine stereotactic radiosurgery (SSRS), also known as spinal radiosurgery, has recently revolutionized the treatment of metastatic spinal disease, offering delivery of ultra-high-dose tumoricidal RT with remarkable accuracy. Local control rates of SSRS approach $90 \%$ up to 2 years after treatment, ${ }^{16,60,61}$ although the accuracy of what is deemed "control" is controversial, given the inability of conventional metrics of tumor size to accurately assess the volume of viable disease and correlate with ultimate tumor progression. ${ }^{43}$

There are no standardized consensus guidelines for tumor imaging after SSRS. There are also few studies evaluating the utility of different imaging modalities after SSRS, none of which use histopathological correlation. Given the lack of data and standardized guidelines, it has been challenging to understand which imaging modalities best assess treatment response, as well as predict toxicity, in these patients. Several challenges present themselves when imaging to assess tumor response after SSRS. Depending on the imaging modality in question, it can at times be difficult to distinguish common treatment effects, such as tumor necrosis and reactive inflammation, from unwanted effects of radiation on the spine. For example, vertebral compression fracture (VCF) occurs in $11 \%-40 \%$ of SSRS sites treated and can be difficult to distinguish from shrinking vertebral body volume due to nonpathologic fracture or reduction in tumor volume..$^{40,42}$

Currently used Spine Response Assessment in NeuroOncology (SPINO) group criteria for defining treatment response in SSRS heavily rely on technically consistent and proper use of imaging to describe morphological changes in treated lesions. ${ }^{47}$ However, these criteria have yet to be validated and are lagging behind experimental advances in current imaging modalities. Advances in imaging may potentially allow the clinician to investigate changes specific to single high-dose radiation treatment and to the type of tumor in question. Thus an understanding of the strengths and weaknesses of each imaging modality in the setting of spinal radiosurgery is required to select the most appropriate imaging for post-treatment monitoring on a clinical basis. In this paper, we review the currently accepted modalities and practice for initial diagnostic assessment of spinal disease and assessment of response to spinal radiosurgery.

\section{Imaging Modalities for Initial Diagnosis of Spine Metastases \\ Computed Tomography}

Due to its high spatial and temporal resolution, CT is more sensitive than conventional radiography for the detection of bone metastases and is often helpful in ruling out metastatic bone disease. CT has shown good correlation with nuclear bone scans for the detection of bone metastases and may even be sufficient if CT of the thorax, abdomen, and pelvis is performed. ${ }^{2} \mathrm{CT}$ can easily characterize lesions as lytic or blastic and may successfully assess paravertebral or intraspinal extensions (Table 1). ${ }^{3}$ This
TABLE 1. Comparison summary of attributes of different imaging modalities utilized in the assessment of metastatic bone disease

\begin{tabular}{lccc}
\hline \multicolumn{1}{c}{ Imaging Modality } & CT & MRI $^{*}$ & $\begin{array}{c}\text { Nuclear } \\
\text { Imaging }\end{array}$ \\
\hline Cost & +++ & ++ & ++ \\
\hline Availability & +++ & ++ & ++ \\
\hline Differentiation btwn lytic \& blastic lesions & +++ & ++ & - \\
\hline Delineation of bony anatomy/fractures & +++ & ++ & - \\
\hline Bone density & +++ & + & - \\
\hline Assessment of marrow \& soft tissue & - & +++ & - \\
\hline Metabolic activity & - & + & +++ \\
\hline Whole body assessment & + & + & +++ \\
\hline
\end{tabular}

+ = poor feasibility; ++ = moderate feasibility; +++ = very feasible; - = not feasible.

* Includes DWI and Gd-enhanced MRI.

information is particularly important prior to interventional therapies such as SSRS. CT also offers significant guidance in assessing bony pathology due to tumor pathology such as VCF and trabecular destruction and can be used to determine risk of pathologic fracture in the future. ${ }^{23}$

\section{Magnetic Resonance Imaging}

MRI is the current gold standard in both the initial detection and morphological characterization of spinal disease. It has a high sensitivity and specificity (80\%-90\% and $90 \%-95 \%$, respectively, in meta-analyses) in detecting malignant skeletal lesions and differentiating them from benign or inflammatory lesions..$^{18}$ MRI is the only modality that directly images the vertebral bone marrow, potentially allowing for earlier detection of disease. It also demonstrates local spread of bone metastases and accurately depicts any extension into the spinal canal when there is concern about posssible cord compression..$^{52}$ There are well-established T1- and T2-weighted changes found with tumor replacement of normal marrow (Fig. 1). ${ }^{38}$ Gadolinium contrast enhancement may be useful for delineating paraspinal disease and accentuating bone marrow abnormalities. The addition of diffusion-weighted imaging (DWI) further enhances tumor delineation by detecting alterations in water mobility and has shown good accuracy in differentiating between metastatic and benign vertebral compression fractures, with one study reporting a positive predictive value of $95 \% .^{1,19,37}$ MRI may also indirectly differentiate between osteoblastic and osteolytic lesions using gradient echo sequences.

There are a variety of initial imaging techniques to assess disease, but T1- and T2-weighted MRI, potentially augmented with short tau inversion recovery (STIR) or DWI, remains the best current method for initial disease assessment. MRI also demonstrates more sensitivity than scintigraphy in detecting bone metastases, likely due to its ability to detect metastatic lesions before changes in bone metabolism allow lesions to be detectable by bone scintigraphy. ${ }^{12,13,15,45}$ It is important to image the entire spine, even in cases of apparently isolated disease, given high rates of concurrent spinal lesions in patients with spinal metastases. ${ }^{25}$ 

A MRI
B MRI
C
CT
T1 pre-contrast
$\mathrm{T} 2$
Myelogram
FDG-
PET/CT
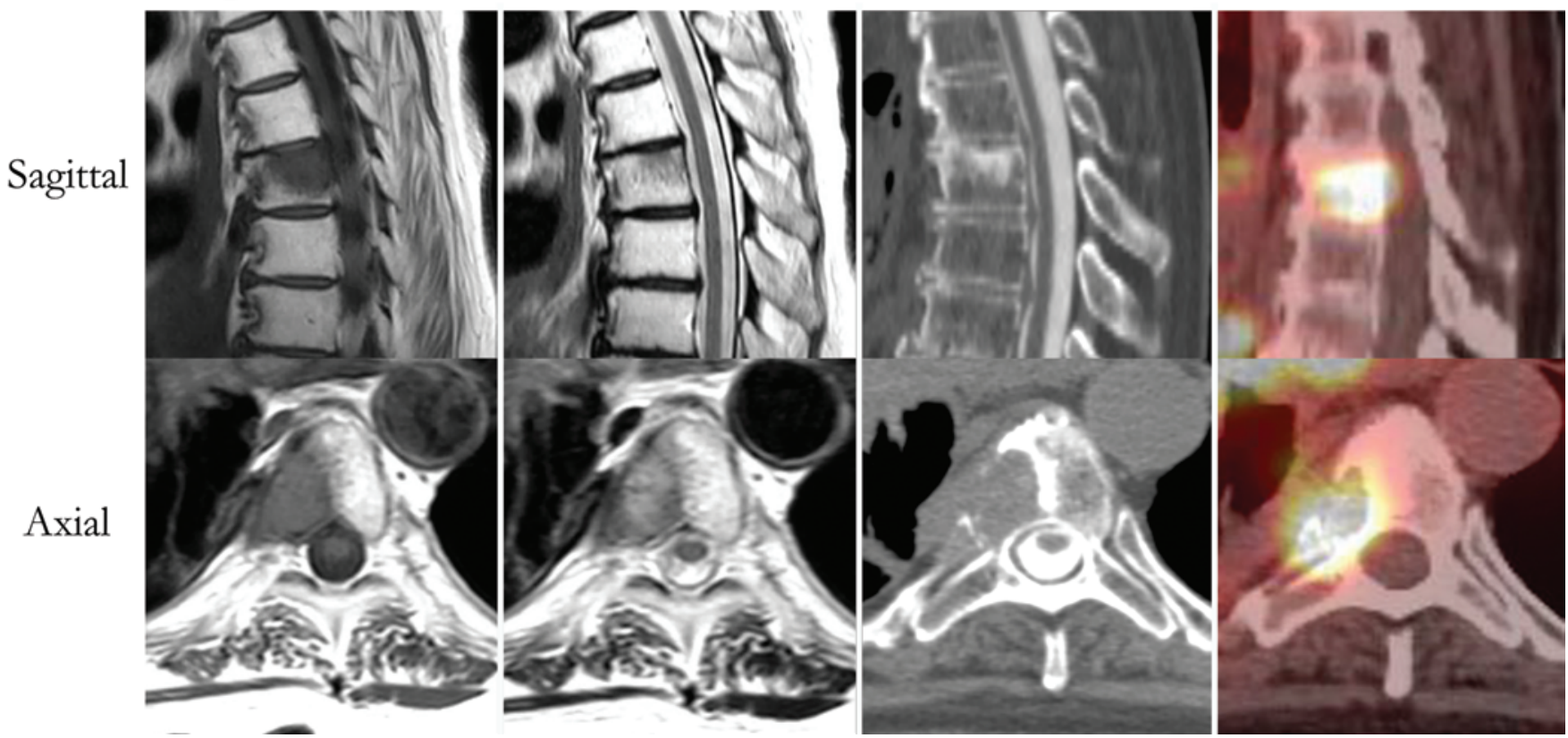

FIG. 1. Images from a representative case illustrating various treatment modalities used to detect spine metastases: T1-weighted pre-contrast MRI (A), T2-weighted MRI (B), CT myelography (C), and FDG PET/CT (D).

\section{Nuclear Imaging and PET}

PET scanning is an alternative means of early detection of malignant bone marrow infiltration, depicting skeletal regions with increased metabolic activity. It has limited spatial resolution in comparison with cross-sectional imaging modalities, such as MRI or CT. There are a number of tracers specific to different tumors or bone-specific tracers. Bone scanning, or bone scintigraphy, utilizes tracers such as ${ }^{99 \mathrm{~m}} \mathrm{Tc}$ or ${ }^{18} \mathrm{~F}$-fluoride to image the secondary bone matrix response that forms secondary to malignant infiltration. Tracers such as ${ }^{18} \mathrm{~F}-\mathrm{FDG}$ exploit the increased glycolytic metabolism of malignant cells to produce increased signal intensity. However, utility of FDG-PET in initial assessment of bone disease may be limited given that osteoblastic tumors tend to have low glucose uptake. ${ }^{32}$ Tracers such as ${ }^{11} \mathrm{C}$-choline and ${ }^{18} \mathrm{~F}$-choline have been shown to offer increased sensitivity in detecting metastatic prostate cancer to the bone, showing potential for early detection of metastatic bone disease and marrow involvement. ${ }^{22}$ PET techniques do offer, however, superior spatial resolution compared with bone scintigraphy and allow determination of standardized uptake values for quantitative longitudinal tumor tracking..$^{51}$

There are a number of tumor-specific tracers that have demonstrated effectiveness in the early detection of spinal cancer. MIBG ( ${ }^{123}$ I-metaiodobenzylguanidine) is one such example and has become standard of care in the evaluation of skeletal involvement in neuroblastoma. Prostatespecific membrane antigen (PSMA) agents for prostate cancer, such as ${ }^{89} \mathrm{Zr}$-J591, outperformed conventional imaging modalities including bone scan and CT in the initial detection of osseous prostate cancer lesions in patients. ${ }^{34}$
Unfortunately, many of these tumor-specific agents remain largely investigational, and much like other antigenspecific compounds, they may be limited by tumoral heterogeneity. Although nuclear medicine techniques allow whole-body imaging, the special considerations of each technique and cost/availability must be accounted for when using imaging.

\section{Imaging in the Evaluation of Spine SSRS Treatment Response \\ Computed Tomography}

CT can provide cortical and trabecular assessment with a high spatial resolution and anatomical detail in relation to the tumor and, unlike MRI, track post-RT changes in bone and mineralization. It is best at evaluating bony structures with accuracy and can readily reveal reossification after successful treatment. ${ }^{53,54}$ Reossification has been shown to occur in response to effective RT in several studies ${ }^{8,21,53-55}$ and to correlate with improvements in symptomatic pain scores. Peri-lesional fibrosis, such as lung fibrosis after thoracic SSRS, can also be easily identified on CT. ${ }^{7}$ Unfortunately, CT is also highly susceptible to artifact from implanted metal hardware. Overall, CT has limited use in assessing treatment response in metastatic soft tissues after SSRS.

\section{Magnetic Resonance Imaging}

For monitoring treatment response after radiation, conventional MRI is the mainstay of imaging, and the most reproducible between institutions, compared with other 
MRI sequences or nuclear imaging techniques. There are well-established T1- and T2-weighted changes that characterize the tumor and later fatty replacement of the normal marrow after RT. ${ }^{38}$ T1-weighted images, augmented by other sequences, allow size and volume assessment of discrete lesions, as well as determination of level of response (i.e., stable or progressive). MRI is the best initial imaging study for spine metastases, provided thin slices are obtained and there is minimal or no skip between slices. ${ }^{10,24,48}$ Vassillou et al. examined 45 patients with bone metastases before and after RT using conventional MRI with contrast and demonstrated significant changes in T1 signal intensity and gadolinium enhancement in patients responding clinically to treatment. ${ }^{53}$ However, conventional MRI provides only anatomical information, and little is gained regarding tumor pathophysiology or viability. Functional and multiparametric MRI techniques can significantly augment the information gained.

Diffusion-weighted MRI accounts for tumor-specific changes in cell density and water diffusion that may occur after radiation treatment. Excluding patients who were treated with bisphosphonates and hydroxyapatite derivatives, Cappabianca et al. used DWI to monitor treatment response after conventionally fractionated RT for bone metastases of multiple histologies. ${ }^{6}$ The group was able to determine post-RT response patterns that were indicative of good response to therapy. Primarily, responders had mean increases in the apparent diffusion coefficient (ADC), due to breakdown of cell membranes and increased water diffusion after RT. Byun et al. had previously found a similar result after RT to only lesions of the spine with quantitative increases in ADC. ${ }^{4}$ However some studies have shown that DWI may not be a suitable modality for monitoring therapeutic response of spinal metastases based on heterogeneity in the ADC changes of the associated malignancies. ${ }^{29,39}$

Dynamic contrast-enhanced MRI (DCE-MRI), or perfusion MRI, offers additional information regarding tumor vascularity but offers debatable diagnostic utility, given limited field of view and substantial institutional variability in perfusion imaging protocols. ${ }^{33}$ DCE-MRI takes advantage of measures of tumor vascularity, such as capillary permeability $\left(\mathrm{k}_{\text {trans }}\right)$ and plasma volume $\left(\mathrm{V}_{\mathrm{p}}\right)$, and potential changes after RT. SSRS is thought, in part, to be effective due to ablation of tumor microvasculature. ${ }^{20} \mathrm{Chu}$ et al. studied 19 lesions with DCE-MRI, with measurements including $\mathrm{k}_{\text {trans }}, \mathrm{V}_{\mathrm{p}}$, area under the curve, and peak enhancement. Plasma volume was the strongest predictor of treatment response, with all treatment successes showing significant decrease in $\mathrm{V}_{\mathrm{p}}$ after $\mathrm{RT}$. These early $\mathrm{V}_{\mathrm{p}}$ decreases were able to predict treatment success in the vast majority of successfully treated tumors. ${ }^{9}$ Spratt et al. studied 12 spine sarcoma metastases treated with SSRS and followed with DCE-MRI. SSRS significantly decreased tumor $\mathrm{V}_{\mathrm{p}}$ and $\mathrm{k}_{\text {trans }}$. Multiparametric MRI utilizing DCE information outperformed clinical radiologists' anatomical impressions of treatment outcomes.$^{43}$ In both radiation and specifically antiangiogenic systemic therapy, vascular changes quantified by DCE-MRI may show predictive changes earlier than morphological changes seen in standard MRI or CT., ${ }^{5,41}$ Based on these results from Spratt et al., the use of multiparametric MRI appears to be the most sensitive imaging technique at detecting response to SSRS and is considered by many to now be the gold standard for monitoring response to treatment. There is currently (at the time of this writing) an ongoing prospective trial evaluating the use of DCE-MRI in the assessment of patients with spinal metastases (NCT02376127; www. clinicaltrials.gov).

It is also important for providers to understand that in the case of post-SSRS follow-up, not only is conventional MRI required, but whole-spine imaging should be undertaken as well. Leeman et al. examined the treatment failure patterns in 88 patients with metastatic sarcoma to the spine and found that the majority of cases of spinal disease progression occur more than 5 vertebral levels away from the index lesion. ${ }^{25}$ There are currently no clear guidelines as to which additional MRI sequences to use in the monitoring of tumor response nor are there criteria defining response in spinal metastases when using techniques such as DCE-MRI. Some significant drawbacks to MRI remain as well, including duration, high cost, and limited availability.

\section{Nuclear Imaging}

In assessing the utility of nuclear medicine techniques for treatment response, we must first distinguish between bone-oriented tracers (e.g., ${ }^{99 \mathrm{~m}} \mathrm{Tc}$ and ${ }^{18} \mathrm{~F}$-fluoride), metabolic tracers (e.g., ${ }^{18} \mathrm{~F}-\mathrm{FDG}$ ), and tumor-directed tracers (e.g., ${ }^{89} \mathrm{Zr}$-J591). Bone-oriented tracers are typically used in bone scans and allow whole-body assessment, but they are subject to many confounding reactions, such as enhanced uptake with hematopoietic growth factors, enhanced uptake during bone remodeling after treatment, and altered uptake after concomitant bisphosphonate uptake. In addition, quantification of metabolic activity for serial assessment is lacking in bone scans. FDG-PET allows for tracking of metabolic activity levels over time, can augment information gleaned from MRI, can overcome implanted metal artifact, and has consensus criteria for reporting of solid lesions. ${ }^{56}$ However, uptake can vary significantly between tumor types (e.g., low FDG uptake in osteoblastic tumors), and FDG and other tracers are subject to "flare"-type reactions secondary to radiation and systemic agents. ${ }^{44}$ In the Response Evaluation Criteria in Solid Tumors (RECIST) version 1.1 criteria, FDG-PET is added only as an adjunct modality to assess disease response and should not be used independently of anatomical imaging (e.g., MRI or CT), if possible. ${ }^{11}$

Nuclear medicine techniques have limited applicability in assessing treatment response in SSRS, depending on the molecular imaging target. It is already understood that bone-directed tracers are inadequate to assess outcomes due to radiation effects on bone remodeling, and metabolic techniques such as FDG-PET should not be used independently of MRI. Targeted molecular imaging with tumor-directed markers attempts to circumvent some of the issues found with other nuclear imaging techniques. By targeting markers specific to the tumor type in question, there is less concern of imaging normal, inflammatory, or bone remodeling reactions. ${ }^{51}$ For example, in an orthotopic bone metastasis mouse model of prostate 
cancer, 89Zr-5A10, which targets prostate specific antigen (PSA), targeted prostate cells without imaging bone remodeling. ${ }^{50}$ In another experimental study, a novel PET tracer targeting the $\alpha v \beta 3$ and $\alpha v \beta 5$ integrins, which are overexpressed in bone metastases, showed rapid and specific uptake into bony metastases in a mouse model. ${ }^{31} \mathrm{Of}$ note, neither of these agents has assessed response after bone-directed RT, but an established tumor-directed tracer for neuroblastoma, MIBG, has shown some utility in monitoring treatment response with decreased signal after SSRS. ${ }^{46}$ Multiple other agents exist or are in development for other tumor-specific markers, such as radiolabeled bombesin analogs against the gastrin-releasing peptide receptor (GRPr), DOTATATE against the somatostatin receptor, and ${ }^{18} \mathrm{~F}$-FES against the estrogen receptor. ${ }^{49,57,58}$ Primary caveats of this approach are both inter- and intratumoral heterogeneity, alteration of expression of molecular targets after any treatment including SSRS, and applicability largely limited to the research environment. Even more of a concern than with MRI, there is the issue of local availability and cost effectiveness with targeted molecular imaging. However, given that targeted imaging avoids the difficulties brought by bone-directed imaging and metabolic imaging, it shows great promise as an option for monitoring treatment response after SSRS.

\section{Discussion and Recommendations}

Several response criteria have been developed to help broadly define tumor response on imaging throughout the body, after radiation or chemotherapy, with examples being the RECIST version 1.1 and MD Anderson (MDA) response criteria. ${ }^{11,17}$ The authors of the RECIST criteria aimed to provide standard assessment of therapeutic response based on definitive changes in tumor size in order to give meaningful comparisons between treatments. However, the criteria left a void in the assessment of spinal and other bony disease, defining osteoblastic lesions and lesions with less than $10 \mathrm{~mm}$ of soft tissue extension as unmeasurable. The MDA criteria set out to fill this void by extending applicability to bone metastases, incorporating CT, MRI, and bone scintigraphy. In a recent study, McDonald et al. found comparable local control rates when comparing RECIST 1.1 size criteria and MDA density criteria in a pool of patients treated with nonspine stereotactic radiosurgery. ${ }^{28}$ There was, however, a significant decrease in local control when using MDA size criteria, although only CT imaging was used in the study and no symptom correlation was performed. Ultimately, neither set of criteria has been validated in the setting of evolving SSRS, and the lack of consistent imaging response criteria remains. Given the revolution and expansion in the multidisciplinary treatment of spine disease, the SPINO group most recently issued guidelines for spinal disease monitoring after SSRS. ${ }^{47}$

The SPINO group formed with the goal of surveying and standardizing reporting of local disease control and pain with imaging methods after SSRS treatment. Local control in spinal radiosurgery was largely defined between institutions as no tumor progression in the SSRS-treated volume or no progression of any treated epidural exten- sion. Nearly all centers imaged patients with MRI 2-3 months after treatment and then 2-6 months thereafter. No institution surveyed routinely used FDG-PET alone in patient follow-up, although some used it as an adjunctive modality for patients with ambiguities in MRI assessment of response. It was also found that few centers use RECIST in the assessment of spinal disease due to poor applicability of their size criteria in bone metastasis. ${ }^{14,47}$ The SPINO group ultimately recommended that MRI be performed for disease monitoring at baseline, 2-3 months after initial treatment for the 1st year, and then every 3-6 months thereafter. They also defined local control as the absence of any definitive progression in the treated region on at least 2 MRI scans performed 6-8 weeks apart and deemed the size-based RECIST version 1.1 as suboptimal. The authors do not report any findings regarding the use of the MDA criteria. Furthermore, the group observed that response criteria in the majority of trials evaluating the effectiveness of spinal radiosurgery were largely inconsistent and thus recommended the development of standardized response criteria in spinal disease.

We acknowledge that future issues to resolve in response assessment is the utility of functional imaging techniques including PET and functional MRI, criteria for assessment of epidural disease, utility of using of RECIST versus developing independent spine criteria, and difficulties in assessing response in the setting of VCF. Further consensus is pending regarding integrating multiparametric MRI and molecular imaging described above into assessing treatment response. SSRS has offered remarkably high rates of local control with limited toxicity to patients with even potentially catastrophic spine disease; however, much is required to determine the best way to assess treatment response in patients and predict who will benefit the most from this treatment. It remains clear that patients should currently be followed with MRI but offered nonstandard imaging techniques in trials when available to further advance our understanding of image-based assessment after SSRS.

\section{Conclusions}

Despite the availability of multiple modalities for imaging and monitoring cancer in the spine, MRI remains essential for assessing treatment response after SSRS for spinal disease, by providing unparalleled morphological information and the highest sensitivity for initial detection of disease. DWI and DCE-MRI can be used to assess treatment response with even higher certainty, allowing longitudinal quantification of pathophysiological changes within tumors, and even allow prognostication of longterm control with imaging biomarkers. FDG-PET has demonstrated utility in showing systemic disease burden and can offer information as an adjunct to MRI but should not be used independently of MRI to assess treatment response. Bone-directed molecular imaging agents alone are not appropriate for monitoring treatment response to SSRS at this time. CT can provide valuable information in the setting of spinal metastasis and SSRS given the risk of VCF and can best differentiate between osteoblastic and osteolytic lesions. Targeted molecular imaging agents 
against tumor-specific markers hold, we believe, great promise for advancing imaging of spine treatment. Current consensus guidelines fully outlining the roles of each of these imaging modalities in SSRS treatment response have yet to be formed.

\section{References}

1. Bonekamp S, Corona-Villalobos CP, Kamel IR: Oncologic applications of diffusion-weighted MRI in the body. J Magn Reson Imaging 35:257-279, 2012

2. Bristow AR, Agrawal A, Evans AJ, Burrell HC, Cornford EJ, James JJ, et al: Can computerised tomography replace bone scintigraphy in detecting bone metastases from breast cancer? A prospective study. Breast 17:98-103, 2008

3. Buhmann Kirchhoff S, Becker C, Duerr HR, Reiser M, BaurMelnyk A: Detection of osseous metastases of the spine: comparison of high resolution multi-detector-CT with MRI. Eur J Radiol 69:567-573, 2009

4. Byun WM, Shin SO, Chang Y, Lee SJ, Finsterbusch J, Frahm $\mathrm{J}$ : Diffusion-weighted MR imaging of metastatic disease of the spine: assessment of response to therapy. AJNR Am J Neuroradiol 23:906-912, 2002

5. Cao Y: The promise of dynamic contrast-enhanced imaging in radiation therapy. Semin Radiat Oncol 21:147-156, 2011

6. Cappabianca S, Capasso R, Urraro F, Izzo A, Raucci A, Di Franco R, et al: Assessing response to radiation therapy treatment of bone metastases: short-term followup of radiation therapy treatment of bone metastases with diffusion-weighted magnetic resonance imaging. J Radiother 2014:698127, 2014

7. Chiu N, Probyn L, Raman S, McDonald R, Poon I, Erler D, et al: Radiological changes on CT after stereotactic body radiation therapy to non-spine bone metastases: a descriptive series. Ann Palliat Med 5:116-124, 2016

8. Chow E, Holden L, Rubenstein J, Christakis M, Sixel K, Vidmar M, et al: Computed tomography (CT) evaluation of breast cancer patients with osteolytic bone metastases undergoing palliative radiotherapy-a feasibility study. Radiother Oncol 70:291-294, 2004

9. Chu S, Karimi S, Peck KK, Yamada Y, Lis E, Lyo J, et al: Measurement of blood perfusion in spinal metastases with dynamic contrast-enhanced magnetic resonance imaging: evaluation of tumor response to radiation therapy. Spine (Phila Pa 1976) 38:E1418-E1424, 2013

10. Costelloe CM, Kundra V, Ma J, Chasen BA, Rohren EM, Bassett RL Jr, et al: Fast Dixon whole-body MRI for detecting distant cancer metastasis: a preliminary clinical study. J Magn Reson Imaging 35:399-408, 2012

11. Eisenhauer EA, Therasse P, Bogaerts J, Schwartz LH, Sargent D, Ford R, et al: New response evaluation criteria in solid tumours: revised RECIST guideline (version 1.1). Eur J Cancer 45:228-247, 2009

12. Eustace S, Tello R, DeCarvalho V, Carey J, Wroblicka JT, Melhem ER, et al: A comparison of whole-body turboSTIR MR imaging and planar 99mTc-methylene diphosphonate scintigraphy in the examination of patients with suspected skeletal metastases. AJR Am J Roentgenol 169:1655-1661, 1997

13. Flickinger FW, Sanal SM: Bone marrow MRI: techniques and accuracy for detecting breast cancer metastases. Magn Reson Imaging 12:829-835, 1994

14. Fournier L, Ammari S, Thiam R, Cuénod CA: Imaging criteria for assessing tumour response: RECIST, mRECIST, Cheson. Diagn Interv Imaging 95:689-703, 2014

15. Frank JA, Ling A, Patronas NJ, Carrasquillo JA, Horvath K, Hickey AM, et al: Detection of malignant bone tumors: MR imaging vs scintigraphy. AJR Am J Roentgenol 155:10431048,1990

16. Gerszten PC, Burton SA, Ozhasoglu C, Welch WC: Ra- diosurgery for spinal metastases: clinical experience in 500 cases from a single institution. Spine (Phila Pa 1976) 32:193-199, 2007

17. Hamaoka T, Madewell JE, Podoloff DA, Hortobagyi GN, Ueno NT: Bone imaging in metastatic breast cancer. J Clin Oncol 22:2942-2953, 2004

18. Heindel W, Gübitz R, Vieth V, Weckesser M, Schober O, Schäfers M: The diagnostic imaging of bone metastases. Dtsch Arztebl Int 111:741-747, 2014

19. Herneth AM, Philipp MO, Naude J, Funovics M, Beichel RR, Bammer R, et al: Vertebral metastases: assessment with apparent diffusion coefficient. Radiology 225:889-894, 2002

20. Kim MS, Kim W, Park IH, Kim HJ, Lee E, Jung JH, et al: Radiobiological mechanisms of stereotactic body radiation therapy and stereotactic radiation surgery. Radiat Oncol J 33:265-275, 2015

21. Koswig S, Budach V: [Remineralization and pain relief in bone metastases after after different radiotherapy fractions (10 times 3 Gy vs. 1 time 8 Gy). A prospective study.] Strahlenther Onkol 175:500-508, 1999 (Ger)

22. Langsteger W, Heinisch M, Fogelman I: The role of fluorodeoxyglucose, ${ }^{18} \mathrm{~F}$-dihydroxyphenylalanine, ${ }^{18} \mathrm{~F}$-choline, and ${ }^{18}$ F-fluoride in bone imaging with emphasis on prostate and breast. Semin Nucl Med 36:73-92, 2006

23. Largo R, Stolzmann P, Fankhauser CD, Poyet C, Wolfsgruber $\mathrm{P}$, Sulser T, et al: Predictive value of low tube voltage and dual-energy CT for successful shock wave lithotripsy: an in vitro study. Urolithiasis 44:271-276, 2016

24. Lecouvet FE, Larbi A, Pasoglou V, Omoumi P, Tombal B, Michoux N, et al: MRI for response assessment in metastatic bone disease. Eur Radiol 23:1986-1997, 2013

25. Leeman JE, Bilsky M, Laufer I, Folkert MR, Taunk NK, Osborne JR, et al: detailed spinal axis patterns of failure following SBRT for metastatic spinal sarcoma. Intern J Rad Oncol 93:E67-E68, 2015

26. Li S, Peng Y, Weinhandl ED, Blaes AH, Cetin K, Chia VM, et al: Estimated number of prevalent cases of metastatic bone disease in the US adult population. Clin Epidemiol 4:87-93, 2012

27. Lutz S, Berk L, Chang E, Chow E, Hahn C, Hoskin P, et al: Palliative radiotherapy for bone metastases: an ASTRO evidence-based guideline. Int J Radiat Oncol Biol Phys 79:965-976, 2011

28. McDonald R, Probyn L, Poon I, Erler D, Brotherston D, Soliman $\mathrm{H}$, et al: Tumor response after stereotactic body radiation therapy to nonspine bone metastases: an evaluation of response criteria. Int J Radiat Oncol Biol Phys 93:879-881, 2015

29. Messiou C, Collins DJ, Giles S, de Bono JS, Bianchini D, de Souza NM: Assessing response in bone metastases in prostate cancer with diffusion weighted MRI. Eur Radiol 21:2169-2177, 2011

30. Moussazadeh N, Laufer I, Yamada Y, Bilsky MH: Separation surgery for spinal metastases: effect of spinal radiosurgery on surgical treatment goals. Cancer Contr 21:168-174, 2014

31. Mühlhausen U, Komljenovic D, Bretschi M, Leotta K, Eisenhut M, Semmler W, et al: A novel PET tracer for the imaging of $\alpha v \beta 3$ and $\alpha v \beta 5$ integrins in experimental breast cancer bone metastases. Contrast Media Mol Imaging 6:413-420, 2011

32. Nakai T, Okuyama C, Kubota T, Yamada K, Ushijima Y, Taniike K, et al: Pitfalls of FDG-PET for the diagnosis of osteoblastic bone metastases in patients with breast cancer. Eur J Nucl Med Mol Imaging 32:1253-1258, 2005

33. Padhani AR, Miles KA: Multiparametric imaging of tumor response to therapy. Radiology 256:348-364, 2010

34. Pandit-Taskar N, O'Donoghue JA, Durack JC, Lyashchenko SK, Cheal SM, Beylergil V, et al: A Phase I/II study for analytic validation of 89Zr-J591 ImmunoPET as a molecular 
imaging agent for metastatic prostate cancer. Clin Cancer Res 21:5277-5285, 2015

35. Patchell RA, Tibbs PA, Regine WF, Payne R, Saris S, Kryscio RJ, et al: Direct decompressive surgical resection in the treatment of spinal cord compression caused by metastatic cancer: a randomised trial. Lancet 366:643-648, 2005

36. Perrin RG, Laxton AW: Metastatic spine disease: epidemiology, pathophysiology, and evaluation of patients. Neurosurg Clin N Am 15:365-373, 2004

37. Pozzi G, Garcia Parra C, Stradiotti P, Tien TV, Luzzati A, Zerbi A: Diffusion-weighted MR imaging in differentiation between osteoporotic and neoplastic vertebral fractures. Eur Spine J 21 (Suppl 1):S123-S127, 2012

38. Ramsey RG, Zacharias CE: MR imaging of the spine after radiation therapy: easily recognizable effects. AJR Am J Roentgenol 144:1131-1135, 1985

39. Reischauer C, Froehlich JM, Koh DM, Graf N, Padevit C, John H, et al: Bone metastases from prostate cancer: assessing treatment response by using diffusion-weighted imaging and functional diffusion maps-initial observations. Radiology 257:523-531, 2010

40. Rose PS, Laufer I, Boland PJ, Hanover A, Bilsky MH, Yamada J, et al: Risk of fracture after single fraction image-guided intensity-modulated radiation therapy to spinal metastases. J Clin Oncol 27:5075-5079, 2009

41. Rosen MA, Schnall MD: Dynamic contrast-enhanced magnetic resonance imaging for assessing tumor vascularity and vascular effects of targeted therapies in renal cell carcinoma. Clin Cancer Res 13:770s-776s, 2007

42. Sahgal A, Whyne CM, Ma L, Larson DA, Fehlings MG: Vertebral compression fracture after stereotactic body radiotherapy for spinal metastases. Lancet Oncol 14:e310-e320, 2013

43. Spratt DE, Arevalo-Perez J, Leeman JE, Gerber NK, Folkert M, Taunk NK, et al: Early magnetic resonance imaging biomarkers to predict local control after high dose stereotactic body radiotherapy for patients with sarcoma spine metastases. Spine J 16:291-298, 2016

44. Stafford SE, Gralow JR, Schubert EK, Rinn KJ, Dunnwald LK, Livingston RB, et al: Use of serial FDG PET to measure the response of bone-dominant breast cancer to therapy. Acad Radiol 9:913-921, 2002

45. Steinborn MM, Heuck AF, Tiling R, Bruegel M, Gauger L, Reiser MF: Whole-body bone marrow MRI in patients with metastatic disease to the skeletal system. J Comput Assist Tomogr 23:123-129, 1999

46. Taunk NK, Kushner B, Ibanez K, Wolden SL: Short-interval retreatment with stereotactic body radiotherapy (SBRT) for pediatric neuroblastoma resulting in severe myositis. Pediatr Blood Cancer 63:731-733, 2016

47. Thibault I, Chang EL, Sheehan J, Ahluwalia MS, Guckenberger M, Sohn MJ, et al: Response assessment after stereotactic body radiotherapy for spinal metastasis: a report from the SPIne response assessment in Neuro-Oncology (SPINO) group. Lancet Oncol 16:e595-e603, 2015

48. Tombal B, Rezazadeh A, Therasse P, Van Cangh PJ, Vande Berg B, Lecouvet FE: Magnetic resonance imaging of the axial skeleton enables objective measurement of tumor response on prostate cancer bone metastases. Prostate 65:178-187, 2005

49. Ulaner GA, Riedl CC, Dickler MN, Jhaveri K, Pandit-Taskar $\mathrm{N}$, Weber W: Molecular Imaging of Biomarkers in Breast Cancer. J Nucl Med 57 (Suppl 1):53S-59S, 2016

50. Ulmert D, Evans MJ, Holland JP, Rice SL, Wongvipat J, Pettersson $\mathrm{K}$, et al: Imaging androgen receptor signaling with a radiotracer targeting free prostate-specific antigen. Cancer Discov 2:320-327, 2012

51. Ulmert D, Solnes L, Thorek DLJ: Contemporary approaches for imaging skeletal metastasis. Bone Res 3:15024, 2015
52. Vassiliou V, Chow E, Kardamakis D: Bone Metastases: A Translational and Clinical Approach. Dordrecht: Springer, 2014

53. Vassiliou V, Kalogeropoulou C, Christopoulos C, Solomou E, Leotsinides M, Kardamakis D: Combination ibandronate and radiotherapy for the treatment of bone metastases: clinical evaluation and radiologic assessment. Int J Radiat Oncol Biol Phys 67:264-272, 2007

54. Vassiliou V, Kalogeropoulou C, Giannopoulou E, Leotsinidis M, Tsota I, Kardamakis D: A novel study investigating the therapeutic outcome of patients with lytic, mixed and sclerotic bone metastases treated with combined radiotherapy and ibandronate. Clin Exp Metastasis 24:169-178, 2007

55. Vassiliou V, Kalogeropoulou C, Mihael L, Tsamandas A, Petsas T, Giannopoulou E, et al: Management of symptomatic bone metastases from breast cancer with concomitant use of external radiotherapy and ibandronate: results of a prospective, pilot study. Breast J 16:92-94, 2010

56. Wahl RL, Jacene H, Kasamon Y, Lodge MA: From RECIST to PERCIST: Evolving considerations for PET response criteria in solid tumors. J Nucl Med 50 (Suppl 1):122S-150S, 2009

57. Wieser G, Mansi R, Grosu AL, Schultze-Seemann W, Dumont-Walter RA, Meyer PT, et al: Positron emission tomography (PET) imaging of prostate cancer with a gastrin releasing peptide receptor antagonist-from mice to men. Theranostics 4:412-419, 2014

58. Wild D, Fani M, Fischer R, Del Pozzo L, Kaul F, Krebs S, et al: Comparison of somatostatin receptor agonist and antagonist for peptide receptor radionuclide therapy: a pilot study. J Nucl Med 55:1248-1252, 2014

59. Wong DA, Fornasier VL, Macnab I: Spinal metastases: the obvious, the occult, and the impostors. Spine (Phila Pa 1976) 15:1-4, 1990

60. Yamada Y, Bilsky MH, Lovelock DM, Venkatraman ES, Toner S, Johnson J, et al: High-dose, single-fraction imageguided intensity-modulated radiotherapy for metastatic spinal lesions. Int J Radiat Oncol Biol Phys 71:484-490, 2008

61. Zelefsky MJ, Greco C, Motzer R, Magsanoc JM, Pei X, Lovelock M, et al: Tumor control outcomes after hypofractionated and single-dose stereotactic image-guided intensity-modulated radiotherapy for extracranial metastases from renal cell carcinoma. Int J Radiat Oncol Biol Phys 82:1744-1748, 2012

\section{Disclosures}

Dr. Kim reports receiving support from EpicentRx for non-studyrelated clinical or research effort.

\section{Author Contributions}

Conception and design: Spratt, Soliman, Taunk, Osborne, Kim, Szerlip. Acquisition of data: all authors. Analysis and interpretation of data: Spratt, Soliman, Taunk, Osborne, Kim, Szerlip. Drafting the article: all authors. Critically revising the article: all authors. Reviewed submitted version of manuscript: all authors. Approved the final version of the manuscript on behalf of all authors: Spratt. Administrative/technical/material support: Spratt, Soliman, Simons, Osborne, Kim, Szerlip. Study supervision: Spratt, Osborne, Kim, Szerlip.

\section{Correspondence}

Daniel E. Spratt, Department of Radiation Oncology, University of Michigan, University Hospital Fl. B2, Rm. C490, 1500 E Medical Center Dr., Ann Arbor, MI 48109. email: sprattda@med. umich.edu. 